\title{
Acute Complicated Pancreatitis With COVID-19 Infection as a Potential Cause
}

\author{
Subhi Mansour ${ }^{\mathrm{a}}$, Kenan Hallon ${ }^{\mathrm{a}}$, Roi Abramov ${ }^{\mathrm{a}}$, Mira Damouny ${ }^{\mathrm{a}}$, Liel Rufeizen ${ }^{\mathrm{a}}$, \\ Rozan Marjiyeh $^{\mathrm{a}}$, Yoram Kluger ${ }^{\mathrm{a}, \mathrm{b}}$, Safi Khuri ${ }^{\mathrm{a}, \mathrm{b}, \mathrm{c}}$
}

\begin{abstract}
The pandemic coronavirus disease 2019 (COVID-19), an infectious disease caused by severe acute respiratory syndrome coronavirus 2 (SARS-CoV-2), has led to a significant and dramatic worldwide increase in morbidity and mortality rates throughout the year 2020. Although most patients present with respiratory symptoms such as cough, fever and shortness of breath, gastrointestinal symptoms have increasingly been reported and recognized as a major element of patients' presentation. It was found that angiotensin-converting enzyme 2 receptors (the entry receptors for SARS-CoV-2) are expressed in the pancreas in addition to the lungs, kidneys and the rest of the gastrointestinal tract, which raises the hypothesis that COVID-19 can stand as an etiology for acute pancreatitis in certain circumstances. Herein, we present a case of a COVID-19 patient who presented to the emergency room with clinical, laboratory and radiological findings of acute pancreatitis several days after being diagnosed with COVID-19 infection. He was admitted and treated conservatively. Several weeks following the initial episode, the patient developed a huge pancreatic pseudocyst, which was treated successfully with endoscopic cystgastrostomy. To the best of our knowledge, following a meticulous review of the current English literature, this is the second case of pancreatic pseudocyst development following acute pancreatitis due to COVID-19 infection.
\end{abstract}

Keywords: Acute pancreatitis; Pancreatic pseudocyst; COVID-19; Endoscopic cyst-gastrostomy; ACE2 receptor

\section{Introduction}

The novel coronavirus disease 2019 (COVID-19) pandemic

Manuscript submitted August 6, 2021, accepted September 23, 2021

Published online November 5, 2021

${ }^{a}$ General Surgery Department, Rambam Health Care Campus, Haifa, Israel ${ }^{b} \mathrm{HPB}$ and Surgical Oncology Unit, Rambam Health Care Campus, Haifa, Israel

${ }^{c}$ Corresponding Author: Safi Khuri, General Surgery Department, Rambam Health Care Campus, Haa'leya Hashniya, Haifa 31096, Israel.

Email: s_khuri@rambam.health.gov.il

doi: https://doi.org/10.14740/jmc3766 poses a great challenge for public health services around the world, due to the fact that it spreads rapidly and causes a significant increase in morbidity and mortality. This virus belongs to a family of single-stranded RNA viruses, which commonly produce respiratory symptoms such as cough, fever and shortness of breath [1]. Gastrointestinal, hepatic and pancreatic involvement has been increasingly reported in several patients, including symptoms such as abdominal pain, diarrhea, nausea, vomiting or gastrointestinal hemorrhage [2]. Abnormal liver function tests and pancreatic enzymes levels were reported in a subset of patients with COVID-19 infection [3, 4]. The expression of angiotensin-converting enzyme 2 (ACE2) receptors in the pancreas raises the possibility of COVID-19-induced pancreatitis. Herein, we present a rare case of COVID-19-induced acute pancreatitis, complicated by a huge pseudocyst formation, in the absence of any known risk factors.

\section{Case Report}

\section{Investigations}

A 47-year-old healthy male patient presented to our emergency department complaining of severe upper abdominal pain of 4 days duration. The pain lasted for 4 days, started abruptly, and was described as diffuse, and radiating to the back. He also suffered from nausea, recurrent vomiting, and reduced appetite. Three days prior to his admission, he was diagnosed with COVID-19 infection by the reverse-transcription polymerase chain reaction (RT-PCR) assay method for severe acute respiratory syndrome coronavirus 2 (SARS-CoV-2) via throat swab. On physical examination upon his admission, the patient's vital signs were within normal limits. An abdominal examination revealed upper abdominal diffuse tenderness with guarding. No abdominal mass was palpated. Digital rectal examination was normal.

\section{Diagnosis}

Complete blood count showed increased white blood cells of $16,000 \times 10^{3} / \mu \mathrm{L}$, with $12 \%$ bands. Liver and kidney function tests were within normal limits. Serum amylase level was 2,708 U/L (normal range $25-125 \mathrm{U} / \mathrm{L}$ ). Amylase level was also highly elevated in urine specimen. An upright abdominal 


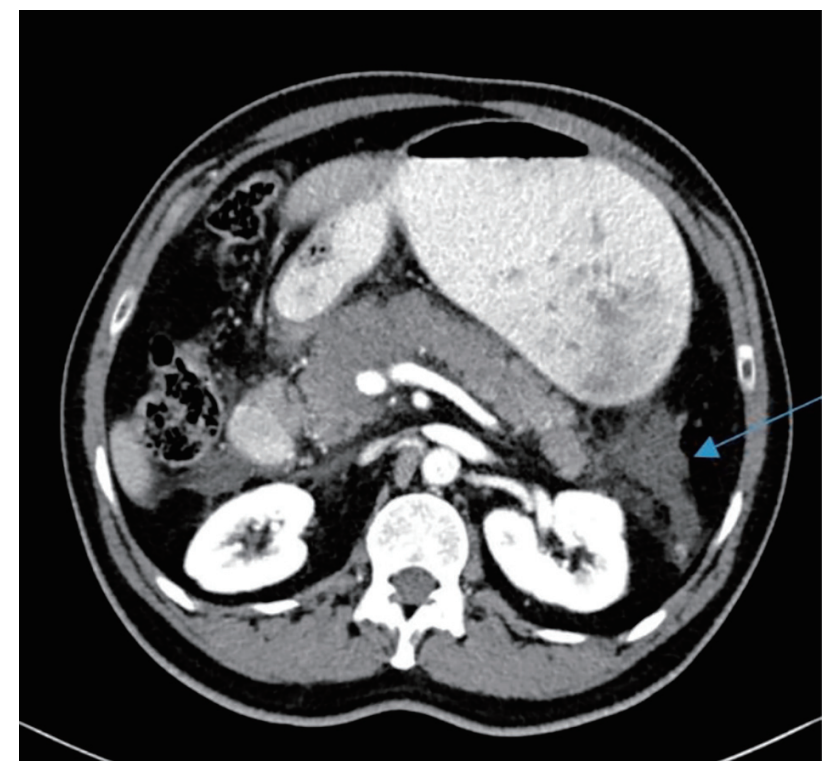

Figure 1. An axial abdominopelvic computed tomography scan revealing an edematous pancreas with ill-defined peripancreatic fluid (arrow)

$\mathrm{X}$-ray and chest X-ray postero-anterior view were normal. A contrast-enhanced computed tomography (CECT) scan of the abdomen and pelvis revealed edema of the pancreas, mainly at the head and body, with inflammatory changes in the pancreas and peripancreatic tissue, as well as ill-defined

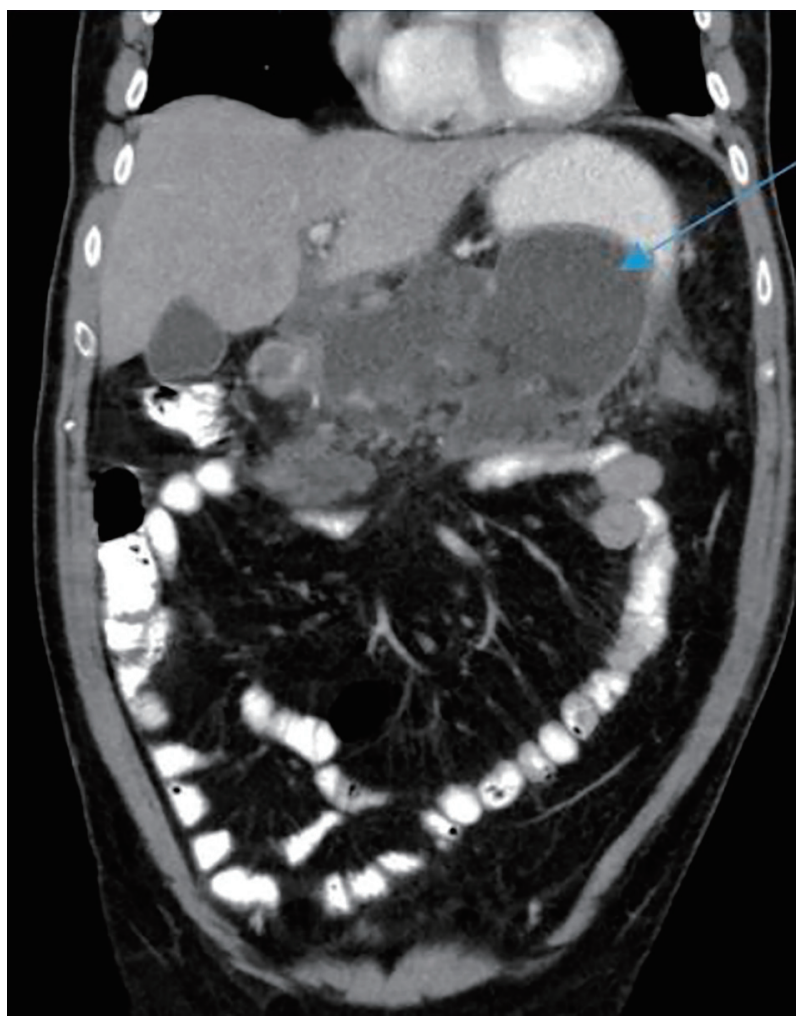

Figure 2. A coronal abdominopelvic computed tomography scan demonstrating multilocular fluid collection around the pancreas (arrow).

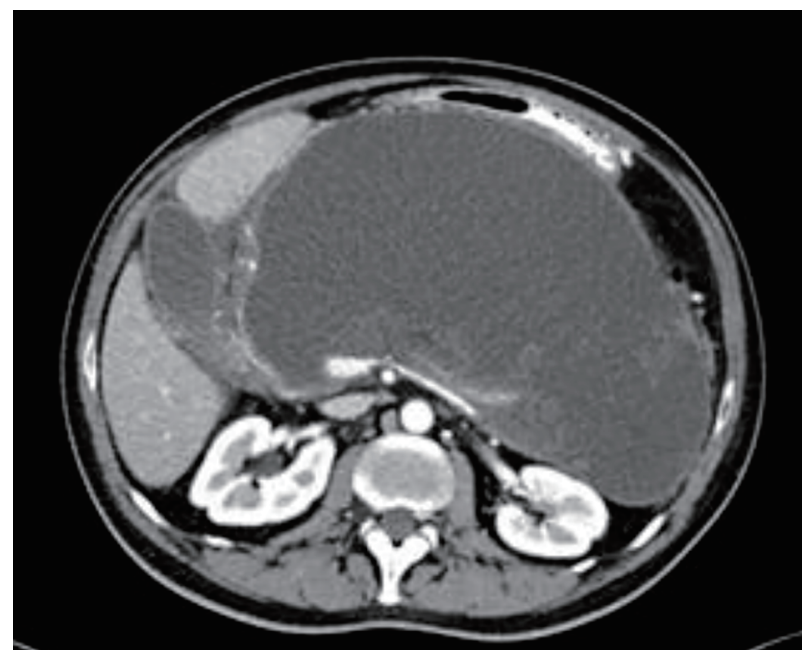

Figure 3. An axial computed tomography scan of the upper abdomen showing a huge pancreatic pseudocyst, with pressure effect on the stomach and duodenum.

peripancreatic fluid (Fig. 1). Multiple subpleural nodules were seen on the lower lobes of bilateral lungs, suggestive of COVID-19 infection. The CT scan findings were consistent with acute pancreatitis grade D according to the CT severity index (CTSI) score.

The patient was admitted with a diagnosis of acute pancreatitis for conservative management by nil per os (NPO) and analgesics. A detailed workup failed to uncover the etiology for the acute pancreatitis. An abdominal ultrasound (USG) for hepatobiliary system was normal and serum calcium, triglycerides and IgG4 levels were within normal limits. The patient negated any history of alcohol usage, recent binge drinking or a recent uptake of new medications. Following conservative management, the patient improved and was discharged on day 4 of his admission. A repeat RT-PCR for SARS-COV-2 via throat swab 10 days following discharge was negative.

Two weeks following his discharge, the patient was readmitted due to upper abdominal pain and fever. A repeat abdominopelvic CT scan revealed edematous pancreas, surrounded by a multilocular fluid collection measuring $21 \times 19$ $\mathrm{cm}$, with a small amount of free intra-abdominal fluid (Fig. 2 ). The patient was once again treated conservatively and discharged home when oral intake was resumed on his third day of admission.

On an outpatient clinic follow-up, 3 weeks later, the patient complained of abdominal distension along with significant increase of abdominal girth, with no nausea, vomiting or abdominal pain. An abdominopelvic CT scan showed a wellcircumscribed fluid collection in the lesser sac measuring 22 $\times 12 \mathrm{~cm}$, causing extrinsic pressure effect on the stomach and duodenum (Fig. 3). These findings were compatible with pancreatic pseudocyst development.

\section{Treatment}

Due to the aforementioned findings, the patient was treated 


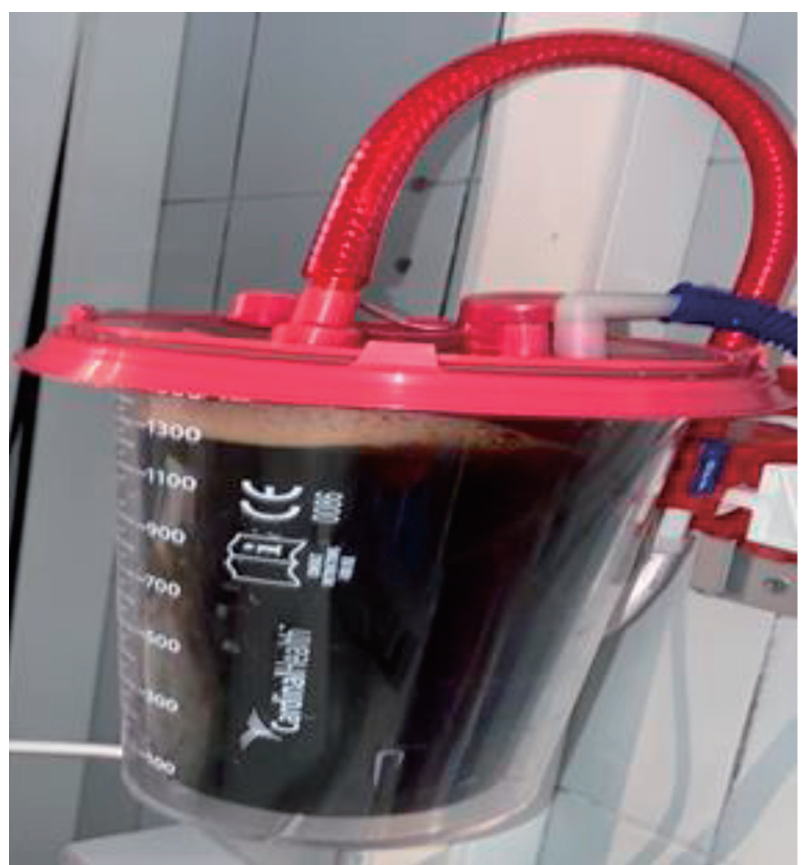

Figure 4. Turbid fluid aspirated before stent insertion.

by NPO, total parenteral nutrition (TPN) and endoscopic cystgastrostomy, by which approximately $4 \mathrm{~L}$ of turbid fluid was aspirated (Fig. 4), and "Hotaxios Boston Lams" stent was inserted (Fig. 5), after which the patient's abdominal distension decreased significantly, and he was discharged with a scheduled repeat CT scan within 3 weeks.

A repeated CT scan of the upper abdomen demonstrated significant improvement in the pancreatic pseudocyst size $(4 \times$ $2.5 \mathrm{~cm}$ ). Five weeks following the endoscopic stent insertion, the patient underwent a scheduled repeat upper endoscopy that revealed an exceedingly small cyst lumen, and the stent was extracted.

\section{Follow-up and outcomes}

On an outpatient clinic follow-up 2 weeks later, the patient was doing well with no gastrointestinal symptoms.

\section{Discussion}

Acute pancreatitis is a common medical reason for hospital admission. Worldwide, the most common cause for acute pancreatitis is gallstone disease, followed by alcohol consumption [5]. Our patient negated alcohol consumption and repeated abdominal USG did not reveal any gallbladder or bile duct stones. Other well-known etiologies of acute pancreatitis include hypertriglyceridemia, hypercalcemia, autoimmune disease and trauma, all of which were excluded in our case. Infectious agents, especially viruses such as hepatitis A and mumps, can also infect the pancreas as reported previously [6]. There were no clinical nor laboratory findings to suggest such an in-

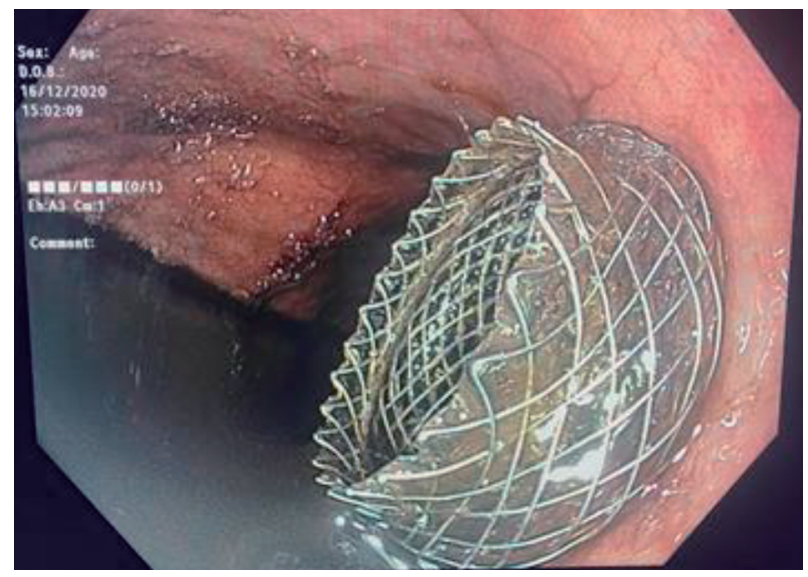

Figure 5. An endoscopic stent insertion through the posterior stomach wall for cyst-gastrostomy.

fection in our patient.

Pancreas injury, defined as high levels of amylase/lipase, was demonstrated in almost $17 \%$ of patients with COVID-19, according to Wang et al [4]. These findings were later questioned as amylase and lipase levels could be elevated due to other disease, such as gastroenteritis. Moreover, gastrointestinal symptoms are eminent COVID-19 symptoms [7], and elevated pancreatic enzymes in COVID-19 patients may not be attributed to acute pancreatitis.

On the other hand, in a retrospective study by Inamadar et al [8], 32 out of 11,883 patients suffered acute pancreatitis, of which $69 \%$ were regarded as having an idiopathic etiology. In addition, Liu et al [9] found that the pancreas could be a target organ for COVID-19 infection, due to the fact that ACE2 receptors are highly expressed in both the exocrine gland, as well as the islet cells of the pancreas, with an even higher concentration than in the lungs. ACE2 receptors act as a receptor for viral entry into the host cells. The previously mentioned findings make SARS-COV-2 a potential etiology for acute pancreatitis.

Although the exact pathogenesis for pancreatitis in SARSCoV-2 is still unclear, two mechanisms were suggested [2]: direct, by cytopathic effects, or indirect systemic inflammatory and immune-mediated cellular response.

Since introducing the hypothesis that COVID-19 infection can induce pancreatitis, several case reports suggesting COVID-19 as an etiology for acute pancreatitis were reported in the English literature during 2020, with a varying degree of severity [10-13].

Pancreatic pseudocyst as a complication of acute pancreatitis in COVID-19 patient was reported by Schepis et al [14], when he detected SARS-CoV-2 RNA in a pancreatic pseudocyst fluid sample collected from a patient with SARS-CoV2-associated pneumonia and a pancreatic pseudocyst developing as a complication of acute edematous pancreatitis. In our case, a sample of the pseudocyst fluid was not sent for SARSCoV-2 RNA testing due to the fact that COVID-19 was not considered as an etiology during the workup.

Herein, we present the second case of pancreatic pseudocyst as a potential complication of COVID-19-induced acute 
pancreatitis, treated successfully by means of endoscopic cystgastrostomy.

\section{Conclusion}

This case report highlights the possibility of COVID-19 infection as a potential cause for acute pancreatitis. RT-PCR of pseudocyst-aspirated sample for SARS-CoV-2 RNA detection is also important in patients who develop acute pancreatitis during COVID-19 infection.

\section{Acknowledgments}

None to declare.

\section{Financial Disclosure}

None to declare.

\section{Conflict of Interest}

The authors have no conflict of interest to declare.

\section{Informed Consent}

Written informed consent was taken from the patient for publication as a case report including relevant images.

\section{Author Contributions}

Acquisition of data and drafting of paper was done by Subhi Mansour, Roi Abramov, Mira Damouny, Liel Rufeizen, Rozan Marjiyeh and Kenan Hallon. Critical revision and final approval of the published version was done by Safi Khuri. All parties agree to be accountable for all aspects of the work in ensuring that questions related to the accuracy or integrity of any part of the work are appropriately investigated and resolved.

\section{Data Availability}

The authors declare that data supporting the findings of this study are available within the article.

\section{References}

1. Guan WJ, Ni ZY, Hu Y, Liang WH, Ou CQ, He JX, Liu L, et al. Clinical characteristics of coronavirus disease 2019 in China. N Engl J Med. 2020;382(18):1708-1720.

2. Patel KP, Patel PA, Vunnam RR, Hewlett AT, Jain R, Jing R, Vunnam SR. Gastrointestinal, hepatobiliary, and pancreatic manifestations of COVID-19. J Clin Virol. 2020;128:104386.

3. Garrido I, Liberal R, Macedo G. Review article: COVID-19 and liver disease-what we know on 1st May 2020. Aliment Pharmacol Ther. 2020;52(2):267-275.

4. Wang F, Wang H, Fan J, Zhang Y, Wang H, Zhao Q. Pancreatic injury patterns in patients with coronavirus disease 19 pneumonia. Gastroenterology. 2020;159(1):367-370.

5. Forsmark CE, Vege SS, Wilcox CM. Acute pancreatitis. N Engl J Med. 2016;375(20):1972-1981.

6. Steinberg W, Tenner S. Acute pancreatitis. N Engl J Med. 1994;330(17):1198-1210.

7. Cheung KS, Hung IFN, Chan PPY, Lung KC, Tso E, Liu $\mathrm{R}, \mathrm{Ng}$ YY, et al. Gastrointestinal manifestations of SARSCoV-2 infection and virus load in fecal samples from a Hong Kong cohort: systematic review and meta-analysis. Gastroenterology. 2020;159(1):81-95.

8. Inamdar S, Benias PC, Liu Y, Sejpal DV, Satapathy SK, Trindade AJ, Northwell C-RC. Prevalence, risk factors, and outcomes of hospitalized patients with coronavirus disease 2019 presenting as acute pancreatitis. Gastroenterology. 2020;159(6):2226-2228 e2222.

9. Liu F, Long X, Zhang B, Zhang W, Chen X, Zhang Z. ACE2 expression in pancreas may cause pancreatic damage after SARS-CoV-2 infection. Clin Gastroenterol Hepatol. 2020;18(9):2128-2130 e2122.

10. Kumaran NK, Karmakar BK, Taylor OM. Coronavirus disease-19 (COVID-19) associated with acute necrotising pancreatitis (ANP). BMJ Case Rep. 2020;13(9):e237903.

11. Alves AM, Yvamoto EY, Marzinotto MAN, Teixeira ACS, Carrilho FJ. SARS-CoV-2 leading to acute pancreatitis: an unusual presentation. Braz J Infect Dis. 2020;24(6):561564.

12. Kataria S, Sharif A, Ur Rehman A, Ahmed Z, Hanan A. COVID-19 induced acute pancreatitis: a case report and literature review. Cureus. 2020;12(7):e9169.

13. Kandasamy S. An unusual presentation of COVID-19: Acute pancreatitis. Ann Hepatobiliary Pancreat Surg. 2020;24(4):539-541.

14. Schepis T, Larghi A, Papa A, Miele L, Panzuto F, De Biase L, Annibale B, et al. SARS-CoV2 RNA detection in a pancreatic pseudocyst sample. Pancreatology. 2020;20(5):1011-1012. 